\title{
Clinical significance of microsurgical excision of the posterior longitudinal ligament using a high-frequency electrosurgical excision procedure in anterior cervical discectomy and fusion
}

\author{
Xiaogang Chen ${ }^{1}$, Guangye Wang ${ }^{1}$, Yuan-Tian Qin ${ }^{2}$, Jin $\mathrm{Li}^{1}, \mathrm{Pu}_{\mathrm{Wang}}{ }^{1}$, Wenjun Huang ${ }^{3}$, Jie Chen ${ }^{1}$ \\ ${ }^{1}$ Department of Orthopedic Surgery, Bao-An District People's Hospital, Shenzhen, China \\ ${ }^{2}$ College of Astronautics, Nanjing University of Aeronautics and Astronautics, Nanjing, China \\ ${ }^{3}$ Department of Orthopedic Surgery, Shenzhen People's Hospital, Shenzhen, China
}

Videosurgery Miniinv 2019; 14 (4): 575-580

DOI: https://doi.org/10.5114/wiitm.2019.84827

\begin{abstract}
Introduction: Anterior cervical decompression and fusion surgery using traditional methods to remove the posterior longitudinal ligament often causes massive bleeding, increasing the risk of surgery. However, the use of a high-frequency electrotome under the microscope can significantly reduce bleeding and reduce the risk of surgery.

Aim: To explore the clinical significance of electrosurgical excision of the posterior longitudinal ligament in the cervical anterior approach under the microscope.

Material and methods: From December 2015 to December 2017, patients who underwent anterior cervical discectomy and fusion at our hospital were followed up. We enrolled 73 men and 50 women who were 30 to 74 years old (mean, 49.96 years). Among 67 patients in group A treated with a high-frequency electrosurgical knife under the microscope, 58 were followed up; among 73 patients in group B treated with a traditional cervical hook knife under the microscope, 65 were followed up. Clinical data, operative time, intraoperative bleeding volume, VAS score, and Japanese Orthopaedic Association (JOA) improvement rate were retrospectively analyzed.

Results: There were significant differences in the mean operative time and intraoperative bleeding volume between the two groups ( $p$ <0.05); however, no significant differences were found in the incidence of cerebrospinal fluid leakage, JOA improvement rate at 3 months postoperatively, and VAS score at 3 months postoperatively between the two groups ( $p>0.05)$.

Conclusions: Electrosurgical resection of the posterior longitudinal ligament of the cervical vertebrae under the microscope can significantly reduce intraoperative bleeding and shorten the operative time and has obvious advantages compared with traditional methods.
\end{abstract}

Key words: cervical spondylosis, anterior approach, electrocautery, posterior longitudinal ligament resection.

\section{Introduction}

Currently, anterior cervical discectomy and fusion (ACDF) is an effective surgical method in the treatment of cervical spondylosis; it always brings about good outcomes and high fusion rates [1, 2]. With regard to surgical treatments for free cervical disc herniation, hypertrophic posterior longitudinal ligament (PLL) cervical spondylosis, and PLL ossification, more researchers advocate that the PLL should be resected during anterior cervical decompression to improve the curative effect [3-6]. However, resection of the PLL during surgery has a risk [7-10].

\section{Address for correspondence}

Guangye Wang, Department of Orthopedic Surgery, Bao-An District People’s Hospital, Shenzhen 518101 Guangdong, China,

e-mail: cnguangyewang@163.com 
Different methods of resection of the PLL are reported. Achieving safe and effective resection of the PLL is a serious issue for experts in this field. With the development of medical technology, anterior cervical nerve decompression assisted by the microscope can result in good surgical results and the refinement of surgery and reduce the incidence of related complications. It has currently become the standard surgical treatment for cervical spondylosis [11-14]. We found that anterior cervical decompression combined with high-frequency electrosurgical resection of the PLL assisted by the microscope can always attain good outcomes and minimal bleeding. This study aimed to explore its clinical value.

\section{Aim}

This study aimed to investigate whether anterior cervical electrotomy of the PLL under the microscope can reduce intraoperative blood loss and shorten operative time, thus proving its superiority over the traditional method of cervical PLL resection.

\section{Material and methods}

\section{Subjects}

Patients who underwent ACDF at our hospital from December 2015 to December 2017 were enrolled. Inclusion criteria were as follows: patients with single- or double-segment cervical spondylotic myelopathy or radiculopathy (cervical disc herniation or PLL hypertrophy, or partial or mild ossification of the PLLt), receiving standard ACDF, and the PLL being excised during surgery. In total, 140 patients were investigated; however, only 123 patients met the inclusion criteria and were enrolled; their mean age was 49.96 years (range: 30-74 years). Sixty-seven patients were treated with a high-frequency electrotome under the microscope, but only 58 patients were followed up (group A). Seventy-three patients were treated with a conventional cervical hook knife, whereas only 65 patients had full follow-up data (group B). All patients underwent anterior and lateral radiography, magnetic resonance imaging, computed tomography, and sagittal reconstruction of the cervical spine and were diagnosed with cervical spondylotic myelopathy or radiculopathy.

This study was conducted in accordance with the Declaration of Helsinki. Approval was obtained from the Ethics Committee of the People's Hospital of Bao'an. Written informed consent was obtained from all patients.

\section{Surgical methods}

All patients underwent surgery in the supine position under general anesthesia. A transverse incision was made at the corresponding interspace to expose the intervertebral fascia. After positioning, the intervertebral fascia was opened, and decompression and fusion were performed under the microscope. For patients in group $A$, the PLL was removed using a high-frequency electrosurgical excision procedure under the microscope. Ultrasound osteotomy was used to remove the bone at the anterior and inferior edges of the upper vertebral body, and the intervertebral disc and end plate of the upper and lower cartilages were scraped. After careful removal of the posterior annulus fibrosus to expose the PLL, refined cauterization of the whole deep PLL was performed with a high-frequency electrotome (Covidien Force FX Electrosurgical Generator, COAG mode and power of $8 \mathrm{~W}$ ). Fine burning excision was performed on the deep layer of the PLL (Photo 1 A). If calcification of the PLL was found, the calcified part was removed using an ultrasound bone knife or a high-speed grinding drill. Subsequently, the transparent superficial PLL was separated and exposed, and the silver-white dura mater was visualized. The superficial PLL was lifted using a nerve dissector and was cut off using a high-frequency electric knife (Photo $1 \mathrm{~B}$ ) until the spinal cord was completely decompressed (Photo $1 \mathrm{C})$. For patients with cervical spondylotic radiculopathy, more attention should be focused on the lateral nerve root foramen. A traditional cervical microneurodissector was used for patients in group $B$. After positioning the deep lateral weakness of the PLL by opening a 4-5-mm incision beside the midline, the PLL was separated from the dural space. If no adhesion was detected, the PLL was removed using a hook knife combined with a laminectomy rongeur. A zero-profile implant was implanted into the intervertebral space in both groups (one ZERO-P BAK) and four internal fixation screws, produced by Johnson \& Johnson, USA). Negative pressure was placed in drains, and the incisions were sutured.

\section{Postoperative management}

The two groups of patients were routinely treated with antibiotics, hormones, dehydrating agents, and 

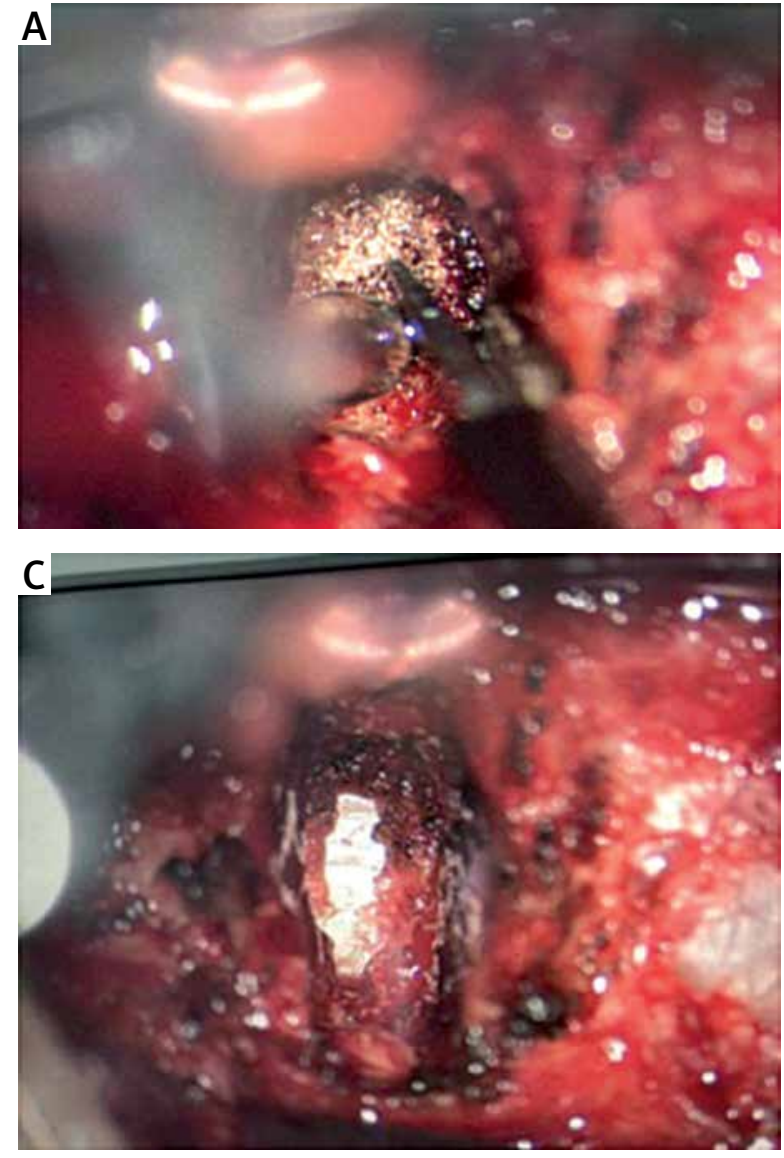

nutritional neurodrugs postoperatively. The drainage tube was removed at 24-48 $\mathrm{h}$ postoperatively, and patients began to practice walking exercises with neck support after $24 \mathrm{~h}$. Follow-up was performed at 3,6 , and 12 months postoperatively to understand the condition of the patient's intervertebral fusion and internal fixator.

\section{Observation index}

The indexes of the two groups were recorded, including the incision length, operative time, bleeding volume, postoperative incision drainage, walking exercise time, and postoperative hospital stay. All patients received their visual pain analog scale (VAS) [15] and Japanese Orthopaedic Association (JOA) score [16] at 3 months postoperatively.

\section{Statistical analysis}

All data are expressed as mean \pm standard deviation. Two independent sample $t$-tests were performed using SPSS 13.0 statistical software

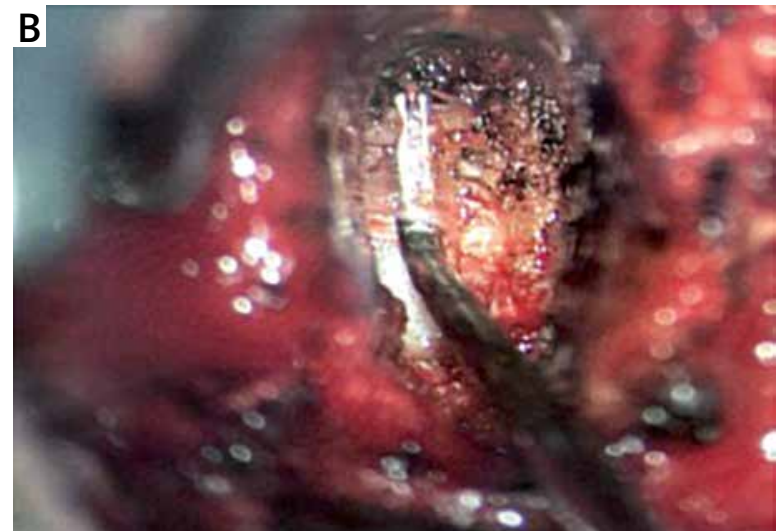

Photo 1. The procedure of PLL removal by high-frequency electrosurgical excision under the microscope. A - Electrosurgical excision of posterior longitudinal ligament; B - isolation of the superficial layer of the posterior longitudinal ligament; $\mathbf{C}$ - exposure of the spinal cord after complete removal of the posterior longitudinal ligament

package to compare differences between the two groups.

\section{Results}

In total, 123 patients were followed up, including 58 and 65 in groups $A$ and $B$, respectively. No significant differences were found in age, sex, disease type, and preoperative JOA scores between the two groups (Table I). In group A, two patients had delayed wound healing; 1 patient had recurrent laryngeal nerve injury after C6/7 discectomy, which recovered spontaneously within 3 weeks. No cerebrospinal fluid leakage after dural injury was found. In group B, 2 patients had delayed wound healing; 1 patient had recurrent laryngeal nerve injury, which recovered spontaneously within 4 weeks. Two patients had intraoperative cerebrospinal fluid leakage due to dural injury and underwent appropriate compression with gelatin sponge intraoperatively. The 2 patients underwent cerebrospinal fluid drainage for $72 \mathrm{~h}$ postoperatively and sandbag compression for 
Table I. Comparisons of general data in the two groups

\begin{tabular}{|lcc|}
\hline Parameters & Group A $(n=58)$ & Group B $(n=65)$ \\
\hline Age [years] & $44.6 \pm 3.96$ & $45.4 \pm 2.43$ \\
\hline Gender: & 33 & 40 \\
\hline Male & 25 & 25 \\
\hline Female & 39 & 40 \\
\hline Types of cervical spondylosis: & 19 & 25 \\
\hline Myelopathy & & 36 \\
\hline Radiculopathy & 31 & 29 \\
\hline Number of operation interspace: & 27 & $12.0 \pm 2.29$ \\
\hline Single & $11.8 \pm 2.33$ & $4.6 \pm 1.74$ \\
\hline Double & $4.47 \pm 1.52$ & \\
\hline Preoperative JOA score & & \\
\hline Preoperative VSA score & & \\
\hline
\end{tabular}

No significant difference was found between the two groups.

Table II. Operation time, bleeding volume and JOA score improvement rate and VAS score of two groups at 3 months after the operation

\begin{tabular}{|lcc|}
\hline Parameter & Group A $(n=58)$ & Group B $(n=65)$ \\
\hline Operation time $[\mathrm{min}]$ & $64.3 \pm 17.51$ & $88.32 \pm 23.28^{*}$ \\
\hline Bleeding volume $[\mathrm{ml}]$ & $15.5 \pm 5.15$ & $55.52 \pm 32.52^{\star}$ \\
\hline VAS score & $3.25 \pm 1.57$ & $3.34 \pm 1.50$ \\
\hline Improvement rate of JOA (\%) & $66.9 \pm 18.70$ & $65.23 \pm 13.64$ \\
\hline
\end{tabular}

Data are shown as mean $\pm S D$. ${ }^{\star} P<0.05$, vs. group $A$.

1 week after drainage tube withdrawal. Both patients underwent primary healing of the incision without lumbar puncture and drainage. Broken cervical disc fragments intruding into the posterior part of the PLL were found in 15 (10.4\%) patients in the two groups. Operative time, intraoperative bleeding volume, and improvement rates of JOA and VAS scores at 3 months postoperatively are shown in Table II. The mean operative time and bleeding volume in group A were significantly lower than those in group $B$ $(p<0.05)$, whereas no significant difference was found in the incidence of cerebrospinal fluid leakage and improvement rate of JOA and VAS scores at 3 months postoperatively $(p>0.05)$.

\section{Discussion}

Hypertrophy of the PLL (HPLL) of the cervical spine is often accompanied by hyperplastic osteophytes.
Formation of osteophytes compresses its adjacent spinal cord and nerve roots. In addition, obvious hyperplasia and hypertrophy can occur in the soft tissue around the osteophyte, particularly the PLL, which is bound to cause fibrous compression $[17,18]$. The compressive range of patients with hyperplasia or hypertrophy of the PLL is larger and more serious than that of those without hyperplasia of the PLL. The degenerated and hypertrophic PLL and its adhesives float forward after decompression by excision of the osteophyte and have limited influence on the decompression effect of osteotomy, which is not conducive to the functional recovery of the compressed spinal cord. The decompression effect of excision of the cervical PLL is positive and conducive to the recovery of spinal morphology and neurological function [19-22].

With the use of surgical instruments and internal fixation materials, the cervical anterior approach 
has been routinely used. However, bleeding problems caused by intravertebral venous plexus injury during the resection of the PLL in anterior cervical surgery often concerns surgeons. Sometimes, larger bleeding volumes hinder surgery, requiring it to be stopped. Cotton sheets and gelatin sponges are mostly used to stop venous plexus bleeding intraoperatively, but the hemostatic effect on patients with severe or multisegmental cervical spondylosis is not satisfactory. Kubo et al. [23] showed that the anterior internal vertebral venous plexus is located in the superficial layer of the PLL rather than the epidural space, and the reasons for the unmanageable intravertebral venous plexus hemorrhage are as follows. The first reason is anatomical characteristics. The anterior venous plexus sneaks in the PLL and clings to the dura mater. During decompression, the longitudinal vessels of the anterior venous plexus are easily damaged. Because blood flow is bidirectional in longitudinal vessels without a venous valve and the vascular wall of the venous plexus is very thin and easily injured, more bleeding occurs if the anterior venous plexus is injured. In addition, the vascular diameter of the cervical intravertebral venous plexus is larger than that of the thoracolumbar vertebral segment [24]. The second reason is the operation scope. If the scope of decompression is relatively narrow, the injured intravertebral venous plexus cannot be exposed to determine the bleeding location. Blind hemostasis not only achieves poor outcomes but also causes more bleeding. The third reason is individual pathological differences, including anatomical differences and differences in patients' conditions. Hemorrhage risk in patients with severe or multisegmental cervical spondylosis, cervical fractures, tumors, or ossification of posterior longitudinal ligament (OPLL) is higher and more serious than that in patients with single-segment cervical spondylosis. Due to long-term compression and compensatory hyperplasia, blood vessels are irregularly and richly distributed, which causes reactive congestion and bleeding during decompression. Therefore, reduction of intravertebral venous plexus hemorrhage is of great importance.

Resection of the PLL with a high-frequency electrotome under the microscope can effectively reduce intraoperative bleeding compared with traditional methods. We summarized the advantages of this method as follows. First, a high-frequency electrotome is handled under the microscope to excise the PLL layer-by-layer; this avoids hemorrhea that may occur in the hook resection of the PLL from both sides. The electrotome can remove the PLL and can synchronously achieve hemostasis. Because it does not require instrument replacement and the removal takes place under the microscope, using the electrotome can achieve almost no bleeding. Second, the surgery is simple, convenient, and practical. Ordinary high-frequency electrotomes can be easily operated at the pure electrocoagulation mode and at a power of $8 \mathrm{~W}$. It does not require any special equipment or professional training. Third, the entire resection process of the PLL with a high-frequency electrotome is basically bloodless and provided a clear surgical field of vision; this greatly reduces the operation of blood suction and saves manpower. It can also avoid the cooperating problem occurring in a narrow space. Fourth, it is safe. During the resection of the PLL, bleeding of the intravertebral venous plexus often obscures the operative field and causes many risks for operators, such as difficulty in finding the bleeding point, cerebrospinal fluid leakage caused by blind hemostasis, sucking blood with a suction apparatus, and spinal cord and nerve root damage. In contrast, these risks can be effectively avoided during electrosurgical excision by ensuring a clear view of the surgical field. Fifth, it can shorten the operative time. Traditional methods often require repeated hemostasis and blood suction with a suction apparatus, whereas a high-frequency electrotome can guarantee a clear surgical field during the entire process of excising the PLL and can save time.

Our study has some limitations. The sample size is small, and the results need to be confirmed in larger cohort studies. Moreover, the superior longterm beneficial effect of microsurgical excision of the PLL by the high-frequency electrosurgical excision procedure in patients with cervical spondylotic myelopathy or radiculopathy compared with traditional methods needs to be further verified.

\section{Conclusions}

In the electrosurgical excision of the PLL in the cervical anterior approach under the microscope, due to the protection of the superficial PLL, the deep PLL can be cauterized with a high-frequency electric knife and the anterior venous plexus can be coagulated to stop bleeding intraoperatively. A clear surgical field can improve the safety of surgery, reduce the rate of repeated operation of surgeons, and shorten the operative time. This surgical method is simple, convenient, practical, and safe. 


\section{Acknowledgments}

This work was supported by the fund from the Science and Technology Project of Shenzhen City, China (Grant No. JCYJ20160427193559599).

\section{Conflict of interest}

The authors declare no conflict of interest.

\section{References}

1. Avila MJ, Skoch J, Sattarov K, et al. Posterior longitudinal ligament resection or preservation in anterior cervical decompression surgery. Clin Neurosci 2015; 22: 1088-90.

2. Schroeder GD, Kurd MF, Millhouse PW, et al. Performing an anterior cervical discectomy and fusion. Clin Spine Surg 2016; 29: 186-90.

3. Wang X, Chen Y, Chen D, et al. Removal of posterior longitudinal ligament in anterior decompression for cervical spondylotic myelopathy. J Spinal Disord Tech 2009; 22: 404-7.

4. Kan SL, Yuan ZF, Ning GZ, et al. Cervical disc arthroplasty for symptomatic cervical disc disease: traditional and Bayesian meta-analysis with trial sequential analysis. Int J Surg 2016; 35 : 111-9.

5. Rao RD, Currier BL, Albert TJ, et al. Degenerative cervical spondylosis: clinical syndromes, pathogenesis, and management. J Bone Joint Surg Am 2007; 89: 1360-78.

6. Bai C, Li K, Guo A, et al. Indication for hypertrophy posterior longitudinal ligament removal in anterior decompression for cervical spondylotic myelopathy. Medicine (Baltimore) 2017; 96: e7043.

7. Kelly MP, Eliasberg CD, Riley MS, et al. Reoperation and complications after anterior cervical discectomy and fusion and cervical disc arthroplasty: a study of 52,395 cases. Eur Spine J 2018; 27: 1432-9.

8. Wang Z, Zhou L, Lin B, et al. Risk factors for non-fusion segment disease after anterior cervical spondylosis surgery: a retrospective study with long-term follow-up of 171 patients. J Orthop Surg Res 2018; 13: 27.

9. Fountas KN, Kapsalaki EZ, Nikolakakos LG, et al. Anterior cervical discectomy and fusion associated complications. Spine (Phila Pa 1976) 2007; 32: 2310-7.

10. Tasiou A, Giannis T, Brotis AG, et al. Anterior cervical spine surgery-associated complications in a retrospective case-control study. J Spine Surg 2017; 3: 444-59.

11. Lei T, Wang H, Tong T, et al. Enlarged anterior cervical diskectomy and fusion in the treatment of severe localised ossification of the posterior longitudinal ligament. J Orthop Surg Res 2016; 11: 129.

12. Maroon JC. Current concepts in minimally invasive discectomy. Neurosurgery 2002; 51: 137-45.

13. Cai RZ, Wang YQ, Wang R, et al. Microscope-assisted anterior cervical discectomy and fusion combined with posterior minimally invasive surgery through tubular retractors for multisegmental cervical spondylotic myelopathy: a retrospective study. Medicine (Baltimore) 2017; 96: e7965.
14. Yang HH, Min JK, Yuan YJ, et al. Anterior cervical intervertebral space decompression under microscope for cervical spondylotic myelopathy in elderly patients. Zhongguo Gu Shang 2015; 28: 36-8.

15. Mc Cormack HM, Horne DJ, Sheather S. Clinical applications of visual analogue scales: a critical review. Psychol Med 1988; 18 : 1007-19.

16. Fujiwara A, Kobayashi N, Saiki K, et al. Association of the Japanese Orthopaedic Association score with the Oswestry Disability Index, Roland-Morris Disability Questionnaire, and shortform 36. Spine (Phila Pa 1976) 2003; 28: 1601-7.

17. Bohlman HH, Emery SE. The pathophysiology of cervical spodylosis and myelopathy. Spine (Phila Pa 1976) 1988; 13: 843-6.

18. Alker G. Neuoradiology of cervical spondylotic myelopathy. Spine (Phila Pa 1976) 1988; 13: 850-3.

19. Missori P, Domenicucci M, Marruzzo D. Clinical effects of posterior longitudinal ligament removal and wide anterior cervical corpectomy for spondylosis. World Neurosurg 2018; 113: e761-8.

20. Mizuno J, Nakagawa H, Song J, Matsuo N. Surgery for dural ossification in association with cervical ossification of the posterior longitudinal ligament via an anterior approach. Neurol India 2005; 53: 354-7.

21. Maharaj MM, Mobbs RJ, Hogan J, et al. Anterior cervical disc arthroplasty (ACDA) versus anterior cervical discectomy and fusion (ACDF): a systematic review and meta-analysis. J Spine Surg 2015; 1: 72-85.

22. Kato S, Murakami H, Demura S, et al. Gradual spinal cord decompression through migration of floated plaques after anterior decompression via a posterolateral approach for OPLL in the thoracic spine. J Neurosurg Spine 2015; 23: 479-83.

23. Kubo Y, Waga S, Kojima T, et al. Microsurgical anatomy of the lower cervical spine and cord. Neurosurgery 1994; 34: 895-900; discussion 901-2.

24. Groen RJ, Groenewegen HJ, van Alphen HA, Hoogland PV. Morphology of the human internal vertebral venous plexus: a cadaver study after intravenous araldite CY 221 injection. Anat Rec 1997; 249: 285-94.

Received: 18.01.2019, accepted: 1.04.2019. 